FORMATION Formation emploi

Revue française de sciences sociales

125 | Janvier-Mars 2014

Pêle-Mêle

\title{
Au sortir du chômage : précaires malgré un contrat à durée indéterminée?
}

Leaving unemployment : being precarious, despite a long term contract

Ausstieg aus der Erwerbslosigkeit : Präkarität trotz unbefristetem

Arbeitsvertrag?

Salida del desempleo : ¿precarios a pesar de un CDI?

Laurence Lizé et Nicolas Prokovas

\section{OpenEdition}

Journals

Édition électronique

URL : http://journals.openedition.org/formationemploi/4154

DOI : 10.4000/formationemploi.4154

ISSN : 2107-0946

Éditeur

La Documentation française

Édition imprimée

Date de publication : 25 mars 2014

Pagination : 89-112

ISSN : 0759-6340

Référence électronique

Laurence Lizé et Nicolas Prokovas, « Au sortir du chômage : précaires malgré un contrat à durée indéterminée? », Formation emploi [En ligne], 125 | Janvier-Mars 2014, mis en ligne le 31 mars 2016, consulté le 30 octobre 2020. URL : http://journals.openedition.org/formationemploi/4154 ; DOI : https://doi.org/10.4000/formationemploi.4154

(c) Tous droits réservés 


\title{
Au sortir du chômage : précaires malgré un contrat à durée indéterminée?
}

\author{
LAURENCE LIZÉ \\ Économiste, maître de conférences, section 5 du CNU (Conseil national des universités). \\ Université de Paris l et Centre d'Économie de la Sorbonne (CES-CNRS)
}

Nicolas Prokovas

Économiste, maître de conférences associé. Université de Paris III, Intégration et Coopération dans l'Espace Européen (ICEE) et Pôle Emploi,

direction des études

Résumé

Au sortir du chômage : précaires malgré un contrat à durée indéterminée ?

Cette étude compare les emplois trouvés par les anciens chômeurs en 2009 à ceux occupés par la population en emploi. Ce segment du marché externe du travail présente des caractéristiques contrastées : certaines sorties du chômage se réalisent dans des conditions $a$ priori favorables (CDI - contrat à durée indéterminée - à temps plein, bien rémunérés, où les personnes se déclarent satisfaites), tandis que d'autres s'effectuent dans un contexte de précarité. Hormis les contrats courts très fréquents, cette précarité concerne aussi une part non négligeable de CDI. Le métier constitue une clé d'entrée pertinente pour appréhender ce phénomène.

Mots clés : travail à durée indéterminée, travail précaire, insertion professionnelle, chômeur

Abstract

Leaving unemployment: being precarious, despite a long term contract

This paper compares jobs occupied by former unemployed to jobs occupied by working people. Some of them leave unemployment in rather avantageous conditions (long term full time contracts, fair salaries, satisfying jobs), whilst others face precariousness. Apart from short term contracts (quite frequent indeed), an important part of long term contracts turns out to be precarious in fact. Analysing the nature of the jobs enables to explain this precariousness.»

Keywords : permanent employment, unstable employment, transition from school to work, unemployed person

Journal of Economic Literature: J 41, J 23

Traduction : Auteurs 
La sortie du chômage entraîne souvent une mobilité professionnelle, parfois contrainte. Certes, le temps passé hors emploi peut avoir été mis à profit pour modifier son profil et son parcours professionnels, notamment par la formation. Cependant, la reprise d'emploi est soumise à la fois aux mutations de l'appareil productif et à des logiques de gestion de l'emploi des entreprises qui, à leur tour, structurent les espaces de mobilité des salariés (Amossé, 2004 ; Dupray, 2005).

Notre étude propose d'observer la segmentation des emplois qui résulte de cette transition à l'œuvre à la sortie du chômage. Elle s'inscrit dans le cadre théorique des approches en termes de segmentation du marché du travail (Doeringer \& Piore, 1971). L'approche ici adoptée privilégie une entrée centrée sur le métier, plus précisément par famille d'activité professionnelle (FAP). Il s'agit en effet d'analyser les contours de la segmentation des emplois à la sortie du chômage sur une année, en 2009. Elle prolonge d'autres travaux qui ont décrit les mobilités professionnelles en termes de métiers (Simmonet \& Ulrich, $2009^{1}$; Lainé, 2010).

La crise a produit des effets différenciés selon les groupes de métiers (Bessière et al., 2010) et ces tendances ressortent dans nos données. Les domaines professionnels qui ont le moins souffert de la récession se trouvent dans le tertiaire : la santé, l'action sociale, les services aux particuliers et aux collectivités et, dans une moindre mesure, le commerce, l'administration et la gestion des entreprises. Il s'agit d'emplois moins sensibles à la conjoncture. Le deuxième groupe concerne des métiers très affectés par la crise mais qui ont rebondi fin 2009, du fait du recours aux contrats courts. Il est composé de métiers industriels, des transports et de la logistique. Sur le troisième groupe de métiers, aucun redressement n'était encore observable en 2009 : les offres d'emploi ont chuté tandis que les demandes augmentaient. Il rassemble les métiers de l'informatique, des télécommunications, les cadres de l'industrie, le bâtiment, l'hôtellerie-restauration, la banque et les assurances.

La crise et la persistance d'un chômage de masse ont tendance à renforcer la sélectivité du marché du travail. Dans ce cadre, la mobilité professionnelle des chômeurs se trouve soumise à des pratiques de recrutement favorisant le développement de l'emploi précaire, à temps partiel ou mal rémunéré. Diverses études révèlent une relation étroite entre la précarité et l'attractivité du secteur professionnel concerné (Givord, 2005 ; Picart, 2008) ; d'autres soulignent que la nature de l'emploi occupé dans le passé (Bruyère \& Lizé, 2010) ou la durée du chômage (Fitoussi et al., 2004) influent sur la mobilité de la main-d'œuvre. En raisonnant en termes de générations, Amossé \& Ben Halima (2010) ont constaté une hausse de la mobilité sur le marché du travail français entre 1982 et 2009 et, dans le même temps, une augmentation de la stabilité dans l'emploi. Ces auteurs démontrent que cet accroissement de la stabilité tient avant tout au vieillissement de certaines générations de la population active ; la dualisation entre le noyau de stabilité et le volant de flexibilité est

1. $30 \%$ des personnes en emploi en 1998 avaient changé de métier en 2003 (Simmonet et Ulrich, 2009). 
en « trompe l'œil » car cette stabilité repose sur trois ou quatre générations de salariés ${ }^{2}$. Le noyau d'emploi très stable s'est constitué autour des recrutements effectués dans les années 1960 et 1970, tandis que le volant de la main-d'œuvre flexible croît de génération en génération.

Avec les départs à la retraite des salariés employés dans des secteurs dotés de marchés internes ou professionnels ${ }^{3}$, la position des nouvelles générations des salariés en contrat permanent risque de se fragiliser et cette dualisation pourrait s'effacer. La structure par âge des sortants de Pôle Emploi semble a priori aller dans le sens de ces analyses : les personnes embauchées sont plus jeunes que la moyenne et ont souvent été recrutées sur des emplois flexibles, soit en termes de type de contrat, soit en termes de durée du travail et, donc, de salaire.

L'analyse par métier des emplois acceptés par les chômeurs permet de repérer certaines formes actuelles de la segmentation de l'emploi. La théorie de la file d'attente et de la concurrence pour l'emploi (Thurow, 1975) est à même de caractériser et d'expliquer le processus d'accès à l'emploi stable, dans la mesure où il n'existe pas de discontinuité marquée entre les individus mais plutôt une organisation continue des flux de main-d'œuvré . Dans cette approche, c'est la nature des emplois à pourvoir qui structure les files d'attente. L'ajustement se réalise ici par les quantités et non par les prix ; les salaires sont ainsi considérés comme fixés préalablement à toute embauche. Par ailleurs, le diplôme n’a pas de valeur intrinsèque ; il sert uniquement de filtre pour sélectionner les candidats qui seront les plus aptes à se former aux besoins de l'entreprise. En ce sens, le rôle du diplôme par rapport à celui des variables caractérisant l'emploi fera l'objet d'une attention particulière. Or, d'après nos données, à la sortie du chômage, les caractéristiques du métier accepté jouent plus que le niveau de formation pour accéder à des emplois pérennes et/ou bien

2. Ce dualisme en «trompe l'ail» s'explique aussi plus largement par le fait que la mobilité tient, pour une part importante, à la progression des passages par le chômage des personnes peu qualifiées (Aeberhardt $\&$ Marbot, 2010). De son côté, la progression de la stabilité dans l'emploi a principalement concerné les postes qualifiés, la fonction publique et les grandes entreprises.

3. Si les mobilités correspondent en majorité à des changements de postes ou des promotions dans l'entreprise, il s'agira plutôt d'emploi du marché interne (Doeringer et Piore, 1971). Les qualifications sont alors peu transférables car acquises par la formation et l'apprentissage sur le tas; en revanche, l'adaptabilité de la main-d'œuvre est forte. Sur ces marchés internes, les trajectoires s'effectuent au sein de l'entreprise ou sur des secteurs d'activité très intégrés, via des compétences spécifiques acquises grâce à une succession d'emplois. Les salaires évoluent principalement en fonction de l'ancienneté dans l'entreprise et de la progression hiérarchique des individus, l'âge intervient donc pour beaucoup. Le marché "professionnel " (Marsden, 1989, 1999 et Eyraud et al., 1990) se caractérise par des possibilités de mobilités horizontales, d'une entreprise ou d'un secteur à l'autre. Avec ou sans diplôme, l'évolution sur les marchés professionnels passe par une période d'apprentissage ; la qualification s'accroît avec l'expérience, grâce aux savoir-faire transmis par les plus anciens ; elle est reconnue par les partenaires sociaux. Ici, la transférabilité des compétences permet des carrières professionnelles plus favorables.

4. Le modèle de la file d'attente est d'ailleurs mobilisé par Doeringer et Piore (1971, p. 169) pour analyser les passages entre les différents marchés. 
rémunérés. En d'autres termes, les caractéristiques individuelles des salariés ne permettent pas, à elles seules, de définir les segments.

Selon l'hypothèse d'une segmentation au niveau des emplois qui complète la vision plus dualiste de la segmentation au niveau de l'entreprise, ce sont les caractéristiques des emplois qui définissent les segments, en partant du principe qu'une même entreprise peut mobiliser de la main-d'œuvre sur différents segments (Piore, 1978 ; Averitt, 1987). Cette vision du marché du travail correspond aussi au modèle de la flexibilité segmentée développé par Gadrey (1992) et par Atkinson (1984) en termes de cœur et de périphérie. Le niveau d'analyse mobilisé ici est celui des emplois, en retenant comme hypothèse qu'une même entreprise peut associer des « mauvais emplois » et des « bons emplois » en son sein.

Les données issues de l'enquête Sortants de Pôle Emploi ne permettent pas de tester toutes les dimensions possibles des différenciations entre les emplois ; par exemple, les conditions de travail ou les possibilités de promotion ne sont pas connues. Les informations qui portent sur le temps de travail, les niveaux de salaire et le degré de stabilité de l'emploi seront ici particulièrement questionnées. La place et le rôle des CDI méritent une attention particulière car ces contrats ne sont pas toujours synonymes d'emploi « de qualité ", en raison notamment de la présence de CDI à temps partiel ou non pérennes.

Après la présentation des données et de la méthode utilisée (partie 1), les caractéristiques des chômeurs qui trouvent un emploi seront confrontées à celles des actifs en emploi (partie 2). Nous interrogerons ensuite la pérennité des emplois retrouvés et la satisfaction des personnes (partie 3). Trois situations seront différenciées : les emplois à temps plein, à temps partiel choisi ou à temps partiel contraint. La possibilité d'être dans l'une ou l'autre de ces situations sera alors évaluée (partie 4).

\section{Données et méthode}

Notre analyse s'appuie sur une exploitation de l'enquête Sortants de Pôle Emploi, complétée par certaines informations tirées du fichier historique des demandeurs d'emploi, de l'enquête Emploi et des DMMO-EMMO (Déclaration des mouvements de maind'œuvre). L’année de référence est 2009, année pendant laquelle les effets de la crise économique se sont fait sentir. Les comparaisons entre les flux de sorties du chômage et les stocks des personnes en emploi sont établies à partir des données disponibles, notamment celles de la Dares reconstituées en FAP (familles professionnelles)5.

5. DARES, Les familles professionnelles - Portraits statistiques 1982 - 2009 (http://travail-emploi.gouv.fr/ etudes-recherche-statistiques-de,76/statistiques, 78/metiers-et-qualifications,83/portraits-statistiquesdes-metiers, 2052/). La nomenclature des FAP est un rapprochement entre la nomenclature des « Professions et catégories socioprofessionnelles » (PCS) utilisée par l'INSEE et le « Répertoire opérationnel des métiers 


\section{Encadré 1 : L'enquête Sortants des listes de Pôle Emploi}

Il s'agit d'une enquête trimestrielle dont chaque vague porte sur 2000 demandeurs d'emploi sortis des listes de Pôle Emploi au cours du dernier mois du trimestre. Les quatre trimestres de l'année 2009 ont été empilés. Le nombre total des personnes ayant déclaré, lors de l'enquête, une sortie des listes pour cause de reprise d'emploi (appelées "sortants du chômage » ou " anciens chômeurs en reprise d'emploi ») s'élève à 4514. Le champ étudié comprend des sorties du chômage sur un statut de salariés (4 407 individus) et d'indépendants (107 individus). Un appariement avec le fichier historique des demandeurs d'emploi a permis d'enrichir les données de l'enquête avec les caractéristiques sociodémographiques de la population étudiée, ainsi qu'avec certaines caractéristiques liées à leur parcours au chômage (ancienneté d'inscription, indemnisation, exercice d'activités réduites).

Cette enquête est réalisée chaque trimestre par téléphone auprès d'un échantillon représentatif de demandeurs d'emploi sortis des catégories $A, B, C^{*}$. Le champ de l'enquête ne comprend que les personnes susceptibles d'être en emploi à la sortie des listes de Pôle Emploi. Ces personnes sont questionnées sur le vrai motif de leur sortie des listes (qui n'avait pas forcément été signalé à Pôle Emploi), sur les conditions de leur retour à l'emploi, sur la nature et le contenu de l'emploi qu'elles ont trouvé, ainsi que sur leur situation professionnelle au moment de l'enquête.

* Il s'agit de demandeurs d'emploi tenus d'entreprendre des actions positives de recherche d'emploi, sans emploi (catégorie A), ayant exercé une activité réduite de 78 heures ou moins au cours du mois (catégorie B) ou ayant exercé une activité réduite de plus de 78 heures au cours du mois (catégorie C).

Dans une première étape, des statistiques descriptives ont été utilisées pour étudier la structure des métiers sur lesquels les chômeurs ont été embauchés. L'objectif est de comparer à la fois les caractéristiques individuelles des sortants du chômage à celles des salariés et les caractéristiques des emplois repris par les anciens chômeurs à celles des emplois en place, notamment en termes de type de contrat, de temps de travail et de salaires pratiqués. Afin d'effectuer ces comparaisons, la clé d'entrée qui semble être la plus pertinente est celle du métier exercé, entendu au sens des FAP (familles professionnelles). En effet, cette nomenclature permet de rapprocher les statistiques de l'emploi (enquête Emploi) et celles du chômage (enquête Sortants de Pôle Emploi et Fichier historique des demandeurs d'emploi).

Les premiers résultats montrent des traits notables : malgré la reprise d'emploi, de nombreuses personnes maintiennent leur inscription à Pôle Emploi, même si l'emploi est en CDI, en déclarant des activités réduites. Par ailleurs, certains CDI semblent fragiles, car $15 \%$ de ces contrats ont duré moins de trois mois. Afin d'expliquer ce résultat, une analyse « toutes choses égales statistiquement par ailleurs " (modèle logit) permet de repérer l'effet propre des variables et d'identifier les facteurs qui jouent sur le risque d'occuper un

et des emplois » (ROME) utilisé par Pôle Emploi ; elle permet ainsi de mettre en regard les statistiques de l'emploi et celles du chômage. 
emploi non pérenne et/ou insatisfaisant. Cette analyse a été ensuite enrichie par un modèle multinomial qui mesure, entre autres, le rôle du CDI dans la probabilité de trouver un emploi à temps plein ou à temps partiel choisi versus un emploi à temps partiel subi.

\section{Structure et caractéristiques des emplois retrouvés par rap- port aux salariés en emploi}

\subsection{Une structure des emplois liée aux métiers, à la taille de l'entreprise et au turnover}

\subsubsection{Une structure des emplois liée aux métiers}

Globalement, 27 familles de métiers concentrent plus de $80 \%$ des flux des sorties du chômage vers l'emploi. Selon l'enquête Emploi, elles représentent $50 \%$ des effectifs en emploi (tableau 1, colonnes a et b). Certaines FAP (agents d'entretien ; vendeurs ; professionnels de l'action sociale, culturelle et sportive) se caractérisent par une forte présence à la fois dans la population active en emploi et parmi les sortants du chômage ; d'autres (enseignants) occupent une place importante dans la structure de la population active en emploi mais pas parmi les sortants du chômage, d'autres encore (employés et agents de maîtrise de l'hôtellerie et de la restauration ; employés administratifs d'entreprise) concentrent une partie relativement importante des sortants du chômage mais pas de la population active en emploi, tandis que douze autres familles (ouvriers qualifiés de l'électricité et de l'électronique ; formateurs ; agents de gardiennage et de sécurité ; bouchers, charcutiers, boulangers...) sont parmi les moins représentées dans les deux populations concernées. Nous centrerons notre analyse sur ces 27 métiers. Il s'agit pour les deux tiers de FAP dont les effectifs ont progressé entre 2000 et 2009 (tableau 1, colonne 1), à un rythme cependant moins soutenu que pour l'ensemble des FAP (5\%). Seuls deux métiers ont connu une augmentation de leurs effectifs supérieure à $30 \%$ pendant la période concernée. Il s'agit des techniciens des services administratifs, comptables et financiers et des aides à domicile et aides ménagères.

\subsubsection{L'impact de la taille de l'entreprise sur la structure des emplois}

Ces tendances sont à mettre en perspective avec la taille de l'entreprise. Pratiquement un emploi sur deux (47\%) s'exerce au sein d'une entreprise de moins de dix salariés ; ce pourcentage dépasse $70 \%$ pour les agriculteurs, les ouvriers qualifiés du gros œuvre du bâtiment, les vendeurs, employés et agents de maîtrise de l'hôtellerie et restauration. L'ancienneté moyenne dans l'emploi est élevée dans certaines familles professionnelles (supérieure ou égale à dix ans pour la moitié des effectifs des enseignants, des agriculteurs, des ouvriers qualifiés du process et de l'électricité, des techniciens de la maintenance et 
des aides-soignants). Dans d'autres métiers, cette ancienneté est faible : elle est inférieure à un an pour plus de $20 \%$ des effectifs des maraîchers-jardiniers, employés administratifs, attachés commerciaux, professionnels de l'action socio-culturelle, caissiers, vendeurs, cuisiniers, employés, et agents de maîtrise de l'hôtellerie et restauration.

\subsubsection{L'influence de la rotation de la main-d'œuvre sur la structure des emplois}

Certains métiers se caractérisent par des taux de rotation de la main-d'œuvre très élevés ; à quelques exceptions près, il s'agit de FAP dont les effectifs ont une faible ancienneté dans l'entreprise. Le taux de rotation comptabilise l'ensemble des flux d'entrées/sorties ; de ce fait, il prend des valeurs bien supérieures à celles du " vrai " turnover (moyenne annuelle des taux entrées/sorties) par FAP, du moins tel que les calculs de la DARES l'avaient fait apparaître pour la période 1998-2002. De surcroît, pour les métiers qui affichent un turnover important, la part des personnes nouvellement recrutées qui se trouvaient auparavant en inactivité ou au chômage est également importante (tableau 1). Ainsi, les FAP où se concentre l'essentiel de la mobilité chômage-emploi et sur lesquelles porte notre analyse, sont celles dont les effectifs se renouvellent plus vite que la moyenne.

Tableau 1. Évolution des effectifs, taux de rotation et ancienneté dans l'entreprise des salariés

\begin{tabular}{|c|c|c|c|c|c|c|c|}
\hline $\begin{array}{c}\text { Familles } \\
\text { professionnelles (FAP) }\end{array}$ & $\begin{array}{c}\% \\
\text { sortants(a) }^{(a)}\end{array}$ & $\begin{array}{c}\% \\
\text { emploi }{ }^{(b)}\end{array}$ & $\begin{array}{l}\text { Évolution } \\
\text { effectifs }^{(1)}\end{array}$ & Rotation ${ }^{(2)}$ & $\begin{array}{c}\text { Anc }<1 \\
\mathrm{an}^{(3)}\end{array}$ & $\begin{array}{l}\text { Tx entrée / } \\
\text { sortie(4) }\end{array}$ & $\begin{array}{l}\text { dt chômeurs } \\
\text { ou inactifs(5) }\end{array}$ \\
\hline $\begin{array}{l}\text { A00-Agriculteurs, éleveurs, } \\
\text { sylviculteurs, bûcherons }\end{array}$ & $1,4 \%$ & $2,1 \%$ & 77 & nd & $11 \%$ & $8,9 \%$ & $3,0 \%$ \\
\hline $\begin{array}{l}\text { A10-Maraîchers, jardiniers, } \\
\text { viticulteurs }\end{array}$ & $2,1 \%$ & $1,2 \%$ & 99 & $136 \%$ & $20 \%$ & $20,1 \%$ & $8,7 \%$ \\
\hline $\begin{array}{l}\text { B20-0uvriers qualifiés du } \\
\text { gros œuvre du bâtiment }\end{array}$ & $3,1 \%$ & $1,6 \%$ & 110 & $21 \%$ & $17 \%$ & $18,2 \%$ & $5,9 \%$ \\
\hline $\begin{array}{l}\text { B40-0uvriers qualifiés du } \\
\text { bâtiment, } 2^{\text {nd }} œ u v r e\end{array}$ & $2,4 \%$ & $2,1 \%$ & 102 & $24 \%$ & $16 \%$ & $17,6 \%$ & $5,3 \%$ \\
\hline $\begin{array}{l}\text { C10-0uvriers qualifiés de } \\
\text { l'électricité et électronique }\end{array}$ & $1,2 \%$ & $0,3 \%$ & 86 & $18 \%$ & $16 \%$ & $11,4 \%$ & $3,7 \%$ \\
\hline $\begin{array}{l}\text { E10-0uvriers qualifiés des } \\
\text { industries de process }\end{array}$ & $2,7 \%$ & $1,2 \%$ & 85 & $30 \%$ & $11 \%$ & $12,8 \%$ & $4,8 \%$ \\
\hline $\begin{array}{l}\text { G10-Techniciens, agents de } \\
\text { maîtrise de la maintenance } \\
\text { et de l'organisation }\end{array}$ & $1,6 \%$ & $1,6 \%$ & 108 & $14 \%$ & $11 \%$ & $10,9 \%$ & $2,6 \%$ \\
\hline $\begin{array}{l}\text { J10-0uvriers qualifiés de la } \\
\text { manutention }\end{array}$ & $5,1 \%$ & $1,8 \%$ & 99 & $20 \%$ & $18 \%$ & $16,2 \%$ & $5,9 \%$ \\
\hline $\begin{array}{l}\text { J30-Conducteurs de } \\
\text { véhicules }\end{array}$ & $3,7 \%$ & $3,0 \%$ & 100 & $36 \%$ & $17 \%$ & $17,7 \%$ & $5,3 \%$ \\
\hline L00-Secrétaires & $1,9 \%$ & $1,8 \%$ & 85 & $25 \%$ & $15 \%$ & $16,7 \%$ & $7,7 \%$ \\
\hline
\end{tabular}




\begin{tabular}{|c|c|c|c|c|c|c|c|}
\hline \multicolumn{8}{|c|}{ Suite Tableau 1} \\
\hline $\begin{array}{l}\text { L10-Employés de la } \\
\text { comptabilité }\end{array}$ & $1,4 \%$ & $1,4 \%$ & 96 & $17 \%$ & $14 \%$ & $17,2 \%$ & $5,4 \%$ \\
\hline $\begin{array}{l}\text { L20-Employés } \\
\text { administratiffs d'entreprise }\end{array}$ & $5,9 \%$ & $1,7 \%$ & 104 & $118 \%$ & $20 \%$ & $22,8 \%$ & $9,4 \%$ \\
\hline $\begin{array}{l}\text { L40-Techniciens des services } \\
\text { administratifs, comptables } \\
\text { et financiers }\end{array}$ & $2,6 \%$ & $1,4 \%$ & 134 & $12 \%$ & $14 \%$ & $13,6 \%$ & $4,0 \%$ \\
\hline $\begin{array}{l}\text { R00-Caissiers, employés de } \\
\text { libre service }\end{array}$ & $2,2 \%$ & $1,2 \%$ & 98 & $152 \%$ & $24 \%$ & $25,1 \%$ & $10,5 \%$ \\
\hline R10-Vendeurs & $6,5 \%$ & $3,3 \%$ & 108 & $66 \%$ & $26 \%$ & $22,2 \%$ & $9,9 \%$ \\
\hline $\begin{array}{l}\text { R20-Attachés commerciaux } \\
\text { et représentants }\end{array}$ & $1,7 \%$ & $2,1 \%$ & 109 & $23 \%$ & $21 \%$ & $21,2 \%$ & $6,9 \%$ \\
\hline $\begin{array}{l}\text { So0-Bouchers, charcutiers, } \\
\text { boulangers }\end{array}$ & $1,0 \%$ & $1,0 \%$ & 96 & $36 \%$ & $19 \%$ & $20,1 \%$ & $4,7 \%$ \\
\hline S10-Cuisiniers & $2,9 \%$ & $1,3 \%$ & 106 & $126 \%$ & $28 \%$ & $24,7 \%$ & $7,3 \%$ \\
\hline $\begin{array}{l}\text { S20-Employés et agents de } \\
\text { maîtrise de l'hôtellerie et de } \\
\text { la restauration }\end{array}$ & $5,1 \%$ & $1,4 \%$ & 109 & $145 \%$ & $37 \%$ & $35,2 \%$ & $14,9 \%$ \\
\hline $\begin{array}{l}\text { T21-Aides à domicile et } \\
\text { aides ménagères }\end{array}$ & $1,5 \%$ & $2,1 \%$ & 151 & $61 \%$ & $17 \%$ & $18,4 \%$ & $10,7 \%$ \\
\hline T22-Assistants maternels & $3,1 \%$ & $1,7 \%$ & 112 & $37 \%$ & $17 \%$ & $18,9 \%$ & $11,1 \%$ \\
\hline $\begin{array}{l}\text { T30-Agents de gardiennage } \\
\text { et de sécurité }\end{array}$ & $1,9 \%$ & $0,8 \%$ & 103 & $80 \%$ & $15 \%$ & $21,9 \%$ & $8,8 \%$ \\
\hline T40-Agents d'entretien & $7,1 \%$ & $4,9 \%$ & 101 & $166 \%$ & $15 \%$ & $16,8 \%$ & $9,2 \%$ \\
\hline V00-Aides-soignants & $1,5 \%$ & $2,1 \%$ & 121 & $146 \%$ & $13 \%$ & $11,2 \%$ & $4,1 \%$ \\
\hline $\begin{array}{l}\text { V40-Professionnels de } \\
\text { l'action sociale, culturelle et } \\
\text { sportive }\end{array}$ & $7,7 \%$ & $2,5 \%$ & 119 & $134 \%$ & $22 \%$ & $21,0 \%$ & $7,1 \%$ \\
\hline W00-Enseignants & $1,5 \%$ & $4,1 \%$ & 100 & nd & $5 \%$ & $11,3 \%$ & $1,9 \%$ \\
\hline W10-Formateurs & $1,2 \%$ & $0,5 \%$ & 128 & nd & $18 \%$ & $18,1 \%$ & $6,7 \%$ \\
\hline Ensemble des FAP & & & 105 & $49 \%$ & $15 \%$ & $16,0 \%$ & $5,8 \%$ \\
\hline
\end{tabular}

Lecture : (a) parmi les emplois retrouvés par les anciens chômeurs, 1,4\% appartiennent à la FAP A00 (agriculteurs...) ; (b) la FAP A00 regroupe 2,1\% de l'ensemble des emplois; (1) indice cumulé de l'évolution des effectifs entre 2000 et 2009, base 100 en 2000. Les effectifs de la FAP A00 ont régressé de $23 \%$ (indice 77) ; (2) taux de rotation des effectifs (somme des taux d'entrée et de sortie) ; (3) ancienneté dans l'entreprise inférieure à 1 an, moyenne de 2007 à 2009. $11 \%$ des salariés de la FAP A00 avaient une ancienneté dans l'entreprise inférieure à 1 an ; (4) moyennes annuelles des taux d'entrée et de sortie entre 1998 et 2002, ce taux était de 8,9\% pour la FAP A00 ; (5) moyennes annuelles des taux d'entrée et de sortie des personnes en provenance du chômage ou de l'inactivité, entre 1998 et 2002. Ce taux était de 3 \% pour la FAP A00.

Sources : (a) enquête Sortants;

(b), (1) et (3) enquête Emploi ;

(2) EMMO-DMMO (données inexistantes pour les FAP A00, W00 et W10) ;

(4) et (5) enquêtes Emploi, traitement DARES (in DARES, Familles professionnelles. Vingt ans de métiers, La Documentation française, 2004, p. 434-435). Les données relatives à la provenance des entrants (notamment de l'inactivité ou du chômage), par famille professionnelle, n'ayant pas fait l'objet d'études plus récentes, nous avons eu recours à une publication antérieure portant sur la période 1998-2002. De ce point de vue, les données des deux dernières colonnes, bien qu'elles ne soient pas strictement comparables à celles des autres colonnes du tableau, apportent des éléments utiles à l'analyse de nos résultats. 


\subsection{Les emplois retrouvés : entre déclassement et précarité}

\subsubsection{Un déclassement à l'embauche}

Si l'on compare certaines caractéristiques des personnes issues du chômage à celles des personnes en emploi au sein des mêmes FAP, on observe que presque partout, la population des sortants du chômage est sensiblement plus jeune et, logiquement, plus formée que celle en emploi, du fait d'un effet de structure par âge marqué.

Cette coexistence entre une main-d'œuvre jeune et diplômée et des emplois peu qualifiés témoigne d'un déclassement à l'embauche - phénomène déjà décrit dans d'autres études (Givord, 2005 ; Lizé \& Prokovas, 2009). Le déclassement semble important dans certaines FAP : celles des aides à domicile et aides ménagères, des agents de gardiennage et de sécurité, des caissiers et employés de libre service, des employés administratifs d'entreprise, des ouvriers qualifiés de l'électricité et de l'électronique.

La structure par sexe ressort moins nettement : certaines FAP, pourtant très féminisées telles que les secrétaires, les caissiers, les vendeurs, ou moyennement féminisées (marầchers-jardiniers, ouvriers qualifiés de l'électricité, cuisiniers), sont relativement moins alimentées par des effectifs féminins en provenance du chômage (tableau 2).

Tableau 2 : Caractéristiques sociodémographiques comparées des salariés en emploi et des anciens chômeurs accédant à l'emploi, par famille professionnelle

\begin{tabular}{|c|c|c|c|c|c|c|}
\hline FAP & Salariés & $\begin{array}{l}\text { Anciens } \\
\text { chômeurs }\end{array}$ & Salariés & $\begin{array}{l}\text { Anciens } \\
\text { chômeurs }\end{array}$ & Salariés & $\begin{array}{l}\text { Anciens } \\
\text { chômeurs }\end{array}$ \\
\hline & \multicolumn{2}{|c|}{ Âge $<25$ ans } & \multicolumn{2}{|c|}{ Diplôme > Bac } & \multicolumn{2}{|c|}{ Part des femmes } \\
\hline $\begin{array}{l}\text { A00-Agriculteurs, } \\
\text { éleveurs, sylviculteurs, } \\
\text { bûcherons }\end{array}$ & $8 \%$ & $37 \%$ & $10 \%$ & $10 \%$ & $28 \%$ & $38 \%$ \\
\hline $\begin{array}{l}\text { A10-Maraîchers, } \\
\text { jardiniers, viticulteurs }\end{array}$ & $14 \%$ & $26 \%$ & $9 \%$ & $16 \%$ & $24 \%$ & $15 \%$ \\
\hline $\begin{array}{l}\text { B20-00 gros œuvre du } \\
\text { bâtiment }\end{array}$ & $11 \%$ & $28 \%$ & $3 \%$ & $9 \%$ & $2 \%$ & $6 \%$ \\
\hline $\begin{array}{l}\text { B40-0Q bâtiment, second } \\
\text { œuvre }\end{array}$ & $10 \%$ & $28 \%$ & $4 \%$ & $7 \%$ & $2 \%$ & $1 \%$ \\
\hline $\begin{array}{l}\text { C10-00 électricité et } \\
\text { électronique }\end{array}$ & $10 \%$ & $29 \%$ & $5 \%$ & $18 \%$ & $34 \%$ & $5 \%$ \\
\hline $\begin{array}{l}\text { E10-0Q industries de } \\
\text { process }\end{array}$ & $7 \%$ & $46 \%$ & $12 \%$ & $9 \%$ & $24 \%$ & $35 \%$ \\
\hline $\begin{array}{l}\text { G10-TAM maintenance et } \\
\text { organisation }\end{array}$ & $8 \%$ & $42 \%$ & $31 \%$ & $44 \%$ & $8 \%$ & $25 \%$ \\
\hline J10-0Q manutention & $7 \%$ & $42 \%$ & $7 \%$ & $10 \%$ & $14 \%$ & $23 \%$ \\
\hline $\begin{array}{l}\text { J30-Conducteurs de } \\
\text { véhicules }\end{array}$ & $5 \%$ & $22 \%$ & $4 \%$ & $8 \%$ & $10 \%$ & $13 \%$ \\
\hline L00-Secrétaires & $7 \%$ & $23 \%$ & $20 \%$ & $36 \%$ & $98 \%$ & $90 \%$ \\
\hline
\end{tabular}




\begin{tabular}{|c|c|c|c|c|c|c|}
\hline \multicolumn{7}{|c|}{ Suite Tableau 2} \\
\hline $\begin{array}{l}\text { L10-Employés de la } \\
\text { comptabilité }\end{array}$ & $9 \%$ & $13 \%$ & $39 \%$ & $50 \%$ & $85 \%$ & $94 \%$ \\
\hline $\begin{array}{l}\text { L20-Employés } \\
\text { administratifs d'entreprise }\end{array}$ & $14 \%$ & $27 \%$ & $29 \%$ & $49 \%$ & $76 \%$ & $83 \%$ \\
\hline $\begin{array}{l}\text { L40-Techniciens des } \\
\text { services administratifs, } \\
\text { comptables et financiers }\end{array}$ & $6 \%$ & $20 \%$ & $48 \%$ & $77 \%$ & $68 \%$ & $66 \%$ \\
\hline $\begin{array}{l}\text { R00-Caissiers, employés } \\
\text { de libre service }\end{array}$ & $24 \%$ & $40 \%$ & $10 \%$ & $24 \%$ & $82 \%$ & $70 \%$ \\
\hline R10-Vendeurs & $23 \%$ & $39 \%$ & $19 \%$ & $24 \%$ & $76 \%$ & $68 \%$ \\
\hline $\begin{array}{l}\text { R20-Attachés } \\
\text { commerciaux et } \\
\text { représentants }\end{array}$ & $10 \%$ & $28 \%$ & $39 \%$ & $60 \%$ & $38 \%$ & $47 \%$ \\
\hline $\begin{array}{l}\text { So0-Bouchers, } \\
\text { charcutiers, boulangers }\end{array}$ & $22 \%$ & $39 \%$ & $4 \%$ & $2 \%$ & $16 \%$ & $20 \%$ \\
\hline S10-Cuisiniers & $21 \%$ & $33 \%$ & $4 \%$ & $8 \%$ & $36 \%$ & $24 \%$ \\
\hline $\begin{array}{l}\text { S20-Employés et AM } \\
\text { hôtellerie-restauration }\end{array}$ & $29 \%$ & $39 \%$ & $13 \%$ & $17 \%$ & $64 \%$ & $62 \%$ \\
\hline $\begin{array}{l}\text { T21-Aides à domicile et } \\
\text { aides ménagères }\end{array}$ & $5 \%$ & $17 \%$ & $4 \%$ & $12 \%$ & $98 \%$ & $98 \%$ \\
\hline T22-Assistants maternels & $6 \%$ & $11 \%$ & $5 \%$ & $9 \%$ & $99 \%$ & $96 \%$ \\
\hline $\begin{array}{l}\text { T30-Agents de } \\
\text { gardiennage et de } \\
\text { sécurité }\end{array}$ & $8 \%$ & $25 \%$ & $8 \%$ & $19 \%$ & $26 \%$ & $33 \%$ \\
\hline T40-Agents d'entretien & $7 \%$ & $27 \%$ & $4 \%$ & $5 \%$ & $70 \%$ & $74 \%$ \\
\hline V00-Aides-soignants & $9 \%$ & $28 \%$ & $7 \%$ & $6 \%$ & $91 \%$ & $90 \%$ \\
\hline $\begin{array}{l}\text { V40-Professionnels de } \\
\text { I'action sociale, culturelle } \\
\text { et sportive }\end{array}$ & $14 \%$ & $27 \%$ & $45 \%$ & $39 \%$ & $66 \%$ & $76 \%$ \\
\hline W00-Enseignants & $2 \%$ & $7 \%$ & $88 \%$ & $90 \%$ & $65 \%$ & $72 \%$ \\
\hline W10-Formateurs & $4 \%$ & $9 \%$ & $55 \%$ & $65 \%$ & $52 \%$ & $55 \%$ \\
\hline Ensemble (toutes FAP) & $9 \%$ & $30 \%$ & $30 \%$ & $23 \%$ & $47 \%$ & $55 \%$ \\
\hline
\end{tabular}

Lecture : parmi les salariés de la famille professionnelle A00 (agriculteurs), $8 \%$ sont âgés de moins de 25 ans, $28 \%$ sont de sexe féminin, $10 \%$ ont un diplôme supérieur au bac ; parmi les anciens chômeurs qui ont trouvé un emploi dans cette famille, 37 \% sont âgés de moins de 25 ans, 38 \% sont de sexe féminin et $10 \%$ possèdent un diplôme supérieur au bac.

Source : enquête Emploi, moyennes annuelles 2007-2009 et enquête Sortants, 2009.

\subsubsection{Un temps partiel subi}

Bien qu'insatisfaisante, la distinction entre le temps partiel choisi et subi a été conservée, d'une part, pour éclairer l'analyse sur les «CDI précaires » en termes de durée du travail et d'autre part, pour montrer les écarts qui existent avec les salariés en emploi. Ici, le temps partiel est considéré comme subi si les personnes ont explicitement déclaré souhaiter travailler davantage. Généralement, le temps partiel subi est sous-estimé dans les enquêtes pour différentes raisons : les personnes qui acceptent un temps partiel n'ont souvent pas le choix et ne le déclarent pas toujours comme " contraint "; elles peuvent aussi déclarer ne 
pas « souhaiter travailler davantage » en raison de difficultés de garde de jeunes enfants par exemple..

Le tableau 3 montre que le temps partiel est généralement bien plus fréquent pour les sortants du chômage que pour les salariés en poste, sauf pour certains métiers tels que les formateurs, les caissiers, les cuisiniers... Pour les anciens chômeurs, ce temps partiel est imposé dans des proportions systématiquement et sensiblement plus élevées que pour les salariés. De ce fait, la qualité des emplois retrouvés par les anciens chômeurs est discutable, conformément à ce qui a déjà été mis en évidence lors de précédents travaux (Lizé \& Prokovas, 2012).

\subsubsection{De faibles rémunérations}

La rémunération obtenue par les sortants du chômage est, pour différentes raisons, très inférieure à celle dont bénéficient les salariés en emploi (tableau 4).

Outre l'ancienneté sur le poste de travail, cet écart s'explique par des modes de gestion de l'emploi différenciés selon les métiers (Chardon \& Estrade, 2007 ; Duhautois et al., 2012). Certaines FAP favorisent plus que d'autres les carrières salariales (techniciens de la maintenance par exemple), d'autres adoptent plutôt des logiques de captation et de rejet ${ }^{6}$ des salariés en pratiquant le salaire minimum horaire en vigueur dans la branche (caissier par exemple).

Compte tenu du rajeunissement et de la féminisation des effectifs issus du chômage, cet écart témoignerait d'un clivage entre les salariés en poste et les nouveaux entrants à l'emploi. Conformément à nos hypothèses, les écarts salariaux, loin d'être uniformes, sont plus ou moins accentués en fonction des familles professionnelles auxquelles appartiennent ces emplois. Ainsi, les écarts se creusent davantage au sein des familles professionnelles dont le salaire médian est plus élevé. Logiquement, ils sont moins importants dans les métiers où les emplois sont les moins bien rémunérés car au niveau du SMIC (salaire minimum interprofessionnel de croissance), tels que assistants maternels, agents de sécurité... Le dernier cas de figure concerne certains métiers " en tension » où les écarts de salaire sont faibles car les entreprises recherchent des candidats. Il s'agit notamment des métiers de bouche (FAP S00, S10), des métiers de l'hôtellerie et de la restauration (S20) et des ouvriers qualifiés du gros œuvre du bâtiment (B20).

6. Ces logiques se réfèrent aux grandes options stratégiques des entreprises en matière de ressources humaines dégagées par Gazier (2010) : la captation de la main-d'œuvre pouvant se faire via des choix de localisation pour des salariés peu qualifiés (embaucher une main-d'œuvre rurale par exemple) ou un avantage salarial différentiel pour attirer les catégories de travailleurs souhaitées. Les modes de rejet des salariés font référence aux différentes options possibles de sortie de l'emploi : fin de contrat à durée limitée, licenciement, rupture conventionnelle ou démission. 


\section{Tableau 3. Emploi à temps partiel des chômeurs qui retrouvent un emploi et des salariés en poste, par ordre (décroissant) d'importance des écarts et par famille professionnelle}

\begin{tabular}{|c|c|c|c|c|}
\hline \multirow[b]{2}{*}{ FAP } & \multicolumn{2}{|c|}{ Salariés } & \multicolumn{2}{|c|}{ Anciens chômeurs } \\
\hline & Temps partiel & dont subi & Temps partiel & dont subi \\
\hline W00-Enseignants & $14 \%$ & $2 \%$ & $48 \%$ & $36 \%$ \\
\hline A10-Maraîchers, jardiniers, viticulteurs & $17 \%$ & $7 \%$ & $44 \%$ & $32 \%$ \\
\hline T40-Agents d'entretien & $37 \%$ & $16 \%$ & $60 \%$ & $41 \%$ \\
\hline L00-Secrétaires & $32 \%$ & $8 \%$ & $54 \%$ & $35 \%$ \\
\hline T30-Agents de gardiennage et de sécurité & $14 \%$ & $6 \%$ & $35 \%$ & $26 \%$ \\
\hline J10-0uvriers qualifiés de la manutention & $4 \%$ & $2 \%$ & $19 \%$ & $14 \%$ \\
\hline T21-Aides à domicile et aides ménagères & $67 \%$ & $26 \%$ & $82 \%$ & $56 \%$ \\
\hline $\begin{array}{l}\text { V40-Professionnels de l'action socioculturelle et } \\
\text { sportive }\end{array}$ & $37 \%$ & $14 \%$ & $52 \%$ & $38 \%$ \\
\hline $\begin{array}{l}\text { L40-Techniciens services admin., comptables, } \\
\text { financiers }\end{array}$ & $15 \%$ & $2 \%$ & $28 \%$ & $13 \%$ \\
\hline L10-Employés de la comptabilité & $23 \%$ & $4 \%$ & $35 \%$ & $29 \%$ \\
\hline T22-Assistants maternels & $33 \%$ & $12 \%$ & $44 \%$ & $27 \%$ \\
\hline J30-Conducteurs de véhicules & $10 \%$ & $4 \%$ & $20 \%$ & $15 \%$ \\
\hline G10-TAM $\left(^{*}\right)$ de la maintenance et de l'organisation & $4 \%$ & $1 \%$ & $13 \%$ & $8 \%$ \\
\hline B40-0uvriers qualifiés du bâtiment, second œuvre & $3 \%$ & $1 \%$ & $11 \%$ & $9 \%$ \\
\hline E10-0uvriers qualifiés des industries de process & $4 \%$ & $2 \%$ & $11 \%$ & $9 \%$ \\
\hline B20-0uvriers qualifiés du gros œuvre du bâtiment & $2 \%$ & $2 \%$ & $8 \%$ & $6 \%$ \\
\hline V00-Aides-soignants & $22 \%$ & $4 \%$ & $28 \%$ & $19 \%$ \\
\hline A00-Agriculteurs, éleveurs, sylviculteurs, bûcherons & $15 \%$ & $4 \%$ & $19 \%$ & $13 \%$ \\
\hline L20-Employés administratifs d'entreprise & $26 \%$ & $7 \%$ & $30 \%$ & $21 \%$ \\
\hline R10-Vendeurs & $30 \%$ & $11 \%$ & $33 \%$ & $20 \%$ \\
\hline SO0-Bouchers, charcutiers, boulangers & $6 \%$ & $1 \%$ & $9 \%$ & $5 \%$ \\
\hline R20-Attachés commerciaux et représentants & $11 \%$ & $3 \%$ & $9 \%$ & $4 \%$ \\
\hline $\begin{array}{l}\text { S20-Employés et AM de I'hôtellerie et de la } \\
\text { restauration }\end{array}$ & $37 \%$ & $17 \%$ & $35 \%$ & $28 \%$ \\
\hline $\begin{array}{l}\text { C10-0uvriers qualifiés de l'électricité et de } \\
\text { l'électronique }\end{array}$ & $5 \%$ & $2 \%$ & $2 \%$ & $2 \%$ \\
\hline S10-Cuisiniers & $21 \%$ & $9 \%$ & $16 \%$ & $13 \%$ \\
\hline R00-Caissiers, employés de libre service & $47 \%$ & $19 \%$ & $39 \%$ & $30 \%$ \\
\hline W10-Formateurs & $33 \%$ & $13 \%$ & $25 \%$ & $18 \%$ \\
\hline Ensemble (toutes FAP - Familles professionnelles)) & $17 \%$ & $5 \%$ & $32 \%$ & $23 \%$ \\
\hline
\end{tabular}

Lecture : parmi les salariés de la famille professionnelle T40 (agent d'entretien), $37 \%$ travaillent à temps partiel ; pour 16\%, il s'agit d'un temps partiel contraint. Parmi les anciens chômeurs qui ont trouvé un emploi dans cette famille, 60 \% travaillent à temps partiel ; pour 41 \%, il s'agit d'un temps partiel contraint.

(*) TAM : techencien et agent de maîtrise.

Ce tableau est structuré en fonction des écarts entre salariés et chômeurs.

Source : enquête Emploi, moyennes annuelles 2007-2009 et enquête Sortants, 2009. 
Tableau 4. Part des salariés et des chômeurs retrouvant un emploi salarié, dont le salaire est inférieur ou égal à $1250 €$

\begin{tabular}{|c|c|c|c|c|}
\hline FAP & Salariés & $\begin{array}{l}\text { Anciens } \\
\text { chômeurs }\end{array}$ & $\begin{array}{l}\text { Écarts (en } \\
\text { points) }\end{array}$ & $\begin{array}{l}\text { Salaire médian } \\
\text { (salariés) }\end{array}$ \\
\hline A00-Agriculteurs, éleveurs, sylviculteurs, bûcherons & $50 \%$ & $60 \%$ & 10 & $1200 €$ \\
\hline A10-Maraîchers, jardiniers, viticulteurs & $55 \%$ & $60 \%$ & 5 & $1200 €$ \\
\hline B20-0uvriers qualifiés du gros œuvre du bâtiment & $19 \%$ & $25 \%$ & 6 & $1500 €$ \\
\hline B40-0uvriers qualifiés du bâtiment, second œuvre & $19 \%$ & $30 \%$ & 11 & $1500 €$ \\
\hline C10-0uvriers qualifiés de l'électricité et de l'électronique & $23 \%$ & $37 \%$ & 14 & $1400 €$ \\
\hline E10-0uvriers qualifiés des industries de process & $18 \%$ & $39 \%$ & 21 & $1500 €$ \\
\hline G10-TAM $\left(^{*}\right)$ de la maintenance et de l'organisation & $8 \%$ & $23 \%$ & 15 & $1800 €$ \\
\hline J10-0uvriers qualifiés de la manutention & $29 \%$ & $59 \%$ & 30 & $1400 €$ \\
\hline J30-Conducteurs de véhicules & $16 \%$ & $37 \%$ & 21 & $1600 €$ \\
\hline L00-Secrétaires & $34 \%$ & $60 \%$ & 26 & $1400 €$ \\
\hline L10-Employés de la comptabilité & $20 \%$ & $59 \%$ & 39 & $1500 €$ \\
\hline L20-Employés administratifs d'entreprise & $34 \%$ & $60 \%$ & 26 & $1400 €$ \\
\hline $\begin{array}{l}\text { L40-Techniciens des services administratifs, comptables et } \\
\text { financiers }\end{array}$ & $8 \%$ & $22 \%$ & 14 & $1800 €$ \\
\hline R00-Caissiers, employés de libre service & $66 \%$ & $71 \%$ & 5 & $1200 €$ \\
\hline R10-Vendeurs & $56 \%$ & $65 \%$ & 9 & $1200 €$ \\
\hline R20-Attachés commerciaux et représentants & $12 \%$ & $49 \%$ & 37 & $1800 €$ \\
\hline S00-Bouchers, charcutiers, boulangers & $30 \%$ & $35 \%$ & 5 & $1400 €$ \\
\hline S10-Cuisiniers & $43 \%$ & $39 \%$ & -4 & $1300 €$ \\
\hline $\begin{array}{l}\text { S20-Employés et agents de maîtrise de l'hôtellerie et de la } \\
\text { restauration }\end{array}$ & $51 \%$ & $54 \%$ & 3 & $1200 €$ \\
\hline T21-Aides à domicile et aides ménagères & $64 \%$ & $92 \%$ & 28 & $1200 €$ \\
\hline T22-Assistants maternels & $74 \%$ & $81 \%$ & 7 & $1000 €$ \\
\hline T30-Agents de gardiennage et de sécurité & $46 \%$ & $47 \%$ & 1 & $1300 €$ \\
\hline T40-Agents d'entretien & $46 \%$ & $61 \%$ & 15 & $1300 €$ \\
\hline V00-Aides-soignants & $17 \%$ & $34 \%$ & 17 & $1500 €$ \\
\hline V40-Professionnels de l'action sociale, culturelle et sportive & $30 \%$ & $50 \%$ & 20 & $1453 €$ \\
\hline W00-Enseignants & $2 \%$ & $48 \%$ & 46 & $2000 €$ \\
\hline W10-Formateurs & $17 \%$ & $36 \%$ & 19 & $1700 €$ \\
\hline Ensemble (toutes FAP - Familles professionnelles)) & $22 \%$ & $48 \%$ & 24 & 1600 \\
\hline
\end{tabular}

Lecture : $19 \%$ des salariés de la famille professionnelle B20 (ouvriers qualifiés du gros œuvre du bâtiment) ont un salaire inférieur à $1200 €$ mensuels; $25 \%$ des anciens chômeurs qui ont trouvé un emploi salarié dans cette famille sont dans ce cas. Le salaire médian dans cette famille est de $1500 €$. Signe éventuel de tensions sur le marché du travail pour les emplois de cuisiniers, la part des anciens chômeurs ayant un salaire inférieur à $1250 €(39 \%)$ est moins importante que celle des salariés en poste (43\%). Cependant, cette répartition s'inverse pour les tranches salariales supérieures ( $42 \%$ contre $30 \%$ respectivement ont un salaire compris entre $1250 €$ et $1500 €$ et $18 \%$ contre $27 \%$ ont un salaire supérieur à $1500 €$ ).

(*) TAM : techniciens et agents de maitrise.

Source : enquête Emploi, moyennes annuelles 2007-2009 et enquête Sortants, 2009. 


\subsubsection{Un taux élevé de CDI « précaires »}

Le dernier point de notre analyse concerne la part des contrats à durée indéterminée dans l'ensemble des embauches des anciens chômeurs. Les données des DMMO et EMMO ne peuvent pas être utilisées ; en effet, d'une part elles sont incomplètes (leur champ couvre l'ensemble des entreprises à l'exception des employeurs particuliers et de l'État) et, d'autre part, elles regroupent l'ensemble des embauches sur un an, y compris les embauches récurrentes, en contrats saisonniers ou intérimaires. Pour disposer d'un point de comparaison, nous avons utilisé l'enquête Emploi et juxtaposé la part des CDI obtenus par les anciens chômeurs à celle de l'ensemble des contrats salariés en stock (tableau 5).

En moyenne, $87 \%$ des salariés avaient un CDI entre 2007 et 2009 ; ce type de contrat ne concerne que $30 \%$ des sortants du chômage qui ont trouvé un emploi salarié. Cependant, ce taux est parfois relativement élevé ; les écarts sont en effet assez disparates selon les familles professionnelles.

Au-delà, il convient d'observer avec précision la nature de ces emplois en CDI. Pour partie, ils correspondent à des CDI proches de la norme d'emploi, à temps plein et bien rémunérés. Ces contrats concernent aussi des métiers en tension, où les employeurs proposent davantage de CDI pour attirer des candidats qui sont au chômage.

Dans une autre configuration, les anciens chômeurs trouvent des CDI plus fréquemment que la moyenne dans des métiers qui se caractérisent par des taux de rotation de la main-d'œuvre très élevés (cuisiniers, aides à domicile, agents de gardiennage et de sécurité... $)^{7}$. De plus, dans certains métiers tels que caissiers ou vendeurs, le temps partiel subi est particulièrement présent parmi les emplois retrouvés.

Peut-on en déduire que certains CDI offerts aux anciens chômeurs sont de moins bonne qualité car à temps partiel contraint ou à très bas salaire ? Ou bien, correspondent-ils à certains modes de gestion de l'emploi qui privilégient les CDI par rapport aux CDD en raison de moindres coûts de rotation (pas de prime de précarité, indemnité de licenciement faible ou nulle avant un an d'ancienneté...) ? En ce sens, Rouxel (2009) a montré qu'environ $10 \%$ des CDI sont des CDI « fragiles ». Dans nos données, cette estimation est même supérieure et avoisine $15 \%$.

7. Cf. tableau 1 . 
Tableau 5. Part des CDI parmi l'ensemble des salariés et parmi les anciens chômeurs par FAP

\begin{tabular}{|c|c|c|}
\hline $\operatorname{FAP}(*)$ & Ensemble des salariés ${ }^{(1)}$ & $\begin{array}{l}\text { Anciens chômeurs en } \\
\text { contrat salarié } \\
\text { (2) }\end{array}$ \\
\hline A00-Agriculteurs, éleveurs, sylviculteurs, bûcherons & $6 \%$ & $23 \%$ \\
\hline A10-Maraîchers, jardiniers, viticulteurs & $16 \%$ & $14 \%$ \\
\hline B20-0uvriers qualifiés du gros œuvre du bâtiment & $66 \%$ & $29 \%$ \\
\hline B40-0uvriers qualifiés du bâtiment, second œuvre & $54 \%$ & $38 \%$ \\
\hline C10-Ouvriers qualifiés de l'électricité et de l'électronique & $87 \%$ & $32 \%$ \\
\hline E10-0uvriers qualifiés des industries de process & $91 \%$ & $24 \%$ \\
\hline $\begin{array}{l}\text { G10-Techniciens, agents de maîtrise de la maintenance et de } \\
\text { l'organisation }\end{array}$ & $91 \%$ & $36 \%$ \\
\hline J10-0uvriers qualifiés de la manutention & $87 \%$ & $27 \%$ \\
\hline J30-Conducteurs de véhicules & $84 \%$ & $50 \%$ \\
\hline L00-Secrétaires & $90 \%$ & $32 \%$ \\
\hline L10-Employés de la comptabilité & $90 \%$ & $42 \%$ \\
\hline L20-Employés administratifs d'entreprise & $83 \%$ & $26 \%$ \\
\hline $\begin{array}{l}\text { L40-Techniciens des services administratifs, comptables et } \\
\text { financiers }\end{array}$ & $92 \%$ & $58 \%$ \\
\hline R00-Caissiers, employés de libre service & $84 \%$ & $40 \%$ \\
\hline R10-Vendeurs & $78 \%$ & $37 \%$ \\
\hline R20-Attachés commerciaux et représentants & $85 \%$ & $67 \%$ \\
\hline S00-Bouchers, charcutiers, boulangers & $55 \%$ & $54 \%$ \\
\hline S10-Cuisiniers & $80 \%$ & $35 \%$ \\
\hline $\begin{array}{l}\text { S20-Employés et agents de maîtrise de l'hôtellerie et de la } \\
\text { restauration }\end{array}$ & $77 \%$ & $31 \%$ \\
\hline T21-Aides à domicile et aides ménagères & $86 \%$ & $43 \%$ \\
\hline T22-Assistants maternels & $81 \%$ & $63 \%$ \\
\hline T30-Agents de gardiennage et de sécurité & $91 \%$ & $38 \%$ \\
\hline T40-Agents d'entretien & $78 \%$ & $29 \%$ \\
\hline V00-Aides-soignants & $87 \%$ & $24 \%$ \\
\hline V40-Professionnels de l'action sociale, culturelle et sportive & $63 \%$ & $\mathbf{2 1 \%}$ \\
\hline W00-Enseignants & $93 \%$ & $26 \%$ \\
\hline W10-Formateurs & $71 \%$ & $49 \%$ \\
\hline Ensemble des FAP & $87 \%$ & $30 \%$ \\
\hline
\end{tabular}

Lecture : $66 \%$ des salariés de la famille professionnelle B20 (ouvriers qualifiés du gros œuvre du bâtiment) avaient un contrat à durée indéterminée ; $29 \%$ des anciens chômeurs qui ont trouvé un emploi salarié dans cette famille ont eu ce type de contrat.

(*) FAP : familles professionnelles.

Source : (1) enquête Emploi (moyennes annuelles 2007-2009) ; (2) enquête Sortants, 2009. 


\section{Pérennité et satisfaction des emplois retrouvés dépendent surtout du métier et de l'indemnisation}

\subsection{Poursuivre sa recherche d'emploi, malgré un CDI}

Afin de répondre à ces interrogations, nous nous sommes intéressés à la pérennité de l'emploi retrouvé par les sortants du chômage, à la satisfaction procurée par cet emploi, ainsi qu'à l'éventuelle poursuite de la recherche d'un autre emploi. Ce dernier indicateur exprime à la fois l'insatisfaction liée à l'emploi trouvé et la manière dont la personne concernée anticipe la stabilité de l'emploi qu'elle a retrouvé.

Aussi est-il étonnant de constater que $30 \%$ des anciens chômeurs embauchés en CDI poursuivaient leur recherche d'emploi (38 \% de l'ensemble des chômeurs en reprise d'emploi, soit un écart somme toute relativement faible).

Si $54 \%$ de ceux qui ont continué leur recherche d'emploi étaient en CDD ou en intérim, $22 \%$ (et même plus de $50 \%$ des ouvriers qualifiés de la manutention et des aides à domicile et aides ménagères) étaient cependant en $\mathrm{CDI}$, ce qui laisse supposer qu'ils n'envisageaient pas leur emploi comme une situation pérenne ou satisfaisante... Trois familles professionnelles concentrent à elles seules le tiers des CDI " mécontents » : agents d'entretien (13\%), assistants maternels (9\%) et employés et agents de maîtrise de l'hôtellerie (9\%).

Les principales raisons évoquées ${ }^{8}$ pour expliquer le maintien de la recherche d'emploi sont la nature et la durée du contrat, $19 \%$ et $24 \%$ du total respectivement ; il s'agit évidemment de personnes n'ayant pas trouvé de CDI. Viennent ensuite le salaire et le temps partiel contraint, avec $13 \%$ du total pour chacun de ces deux motifs, pour lesquels les emplois en CDI sont au moins aussi nombreux que les autres.

Par ailleurs, 18 \% des anciens chômeurs en reprise d'emploi se trouvaient sans emploi au moment de l'enquête, soit près de deux mois après leur sortie du chômage (dont $35 \%$ ne se sont même pas réinscrits à Pôle Emploi, signe évident de leur découragement). Parmi eux, $16 \%$ avaient un emploi en CDI, tandis que $63 \%$ avaient un emploi en CDD ou un emploi intérimaire. Cette reprise d'emploi s'est avérée particulièrement fragile pour un tiers des ouvriers qualifiés du gros ouvre du bâtiment et des ouvriers qualifiés de la manutention.

Enfin, parmi les anciens chômeurs qui ont trouvé un emploi, 10 \% avaient déjà changé d'emploi au moment de l'enquête, soit près de trois mois plus tard, en moyenne ; pour $19 \%$ d'entre eux, l'emploi retrouvé était cependant en CDI. De plus, lors de

8. Raisons d'insatisfaction : un seul choix possible dans le questionnaire. 
l'enquête, 10 \% des sortants avec un CDI étaient à nouveau au chômage et $5 \%$ avaient un emploi différent de celui qu'ils occupaient à la sortie des listes de Pôle Emploi. Ces données attestent que le CDI ne suffit pas à fidéliser la main-d'œuvre.

\subsection{Les métiers retrouvés pèsent sur les risques d'insatisfaction et d'emploi non pérenne}

Les raisons d'insatisfaction déclarées dans l'enquête Sortants permettent de comprendre pourquoi l'emploi retrouvé ne correspond pas aux attentes des anciens chômeurs. L'inadéquation avec le niveau de formation, l'expérience et l'insatisfaction en termes de salaire s'avère assez proche, que les contrats soient en CDD/intérim ou en CDI. Les écarts ressortent surtout au niveau du métier, l'insatisfaction est nettement plus forte sur les contrats courts (tableau 6). Dans certaines FAP (ouvriers de la manutention, caissiers-employés de libre service, employés de l'hôtellerie-restauration, agents d'entretien), les taux d'insatisfaction avoisinent voire dépassent les $50 \%$, aussi bien pour les emplois en CDI que pour les emplois en contrat précaire, quel que soit le motif d'insatisfaction?.

Tableau 6 : Motifs d'insatisfaction concernant l'emploi retrouvé, en fonction du contrat, par FAP

\begin{tabular}{|l|c|c|c|}
\hline & $\begin{array}{c}\text { Inadéquation avec le niveau de } \\
\text { formation ou l'expérience }\end{array}$ & Insatisfaction en termes de salaire & Métier non souhaité \\
\hline CDI & $20 \%$ & $27 \%$ & $24 \%$ \\
\hline CDD et intérim & $24 \%$ & $26 \%$ & $35 \%$ \\
\hline
\end{tabular}

Source : enquête Sortants 2009.

FAP: Familles professionnelles.

Deux modèles de régression logistique (logit) permettent d'évaluer les probabilités d'obtenir un emploi "non pérenne " (modèle 1) ou de ne pas être satisfait de l'exercice de l'emploi trouvé (modèle 2). Ces deux paramètres ont été pris en compte dans leur dimension "objective " et ne se basent pas sur les déclarations des personnes interrogées.

Ainsi, la "non-pérennité " de l'emploi correspond aux situations où l'emploi avait cessé d'exister au moment de l'enquête (indépendamment du contrat formel de travail, c'est-à-dire y compris pour les emplois en CDI ou en CDD de plusieurs mois).

L' "insatisfaction " liée à l'emploi renvoie aux cas où, malgré la reprise d'emploi, la personne a maintenu sa recherche d'emploi, nonobstant une appréciation globalement

9. À l'exception, bien logiquement, des motifs liés au type et à la durée du contrat du travail. 
positive qu'elle peut porter sur l'emploi retrouvé et qui, à notre sens, traduit davantage le fait d'être sorti du chômage que d'avoir trouvé cet emploi précis.

Dans les deux modèles, les variables individuelles jouent moins fortement que celles liées à l'emploi ; en d'autres termes, les métiers acceptés par les anciens chômeurs pèsent plus sur les risques d'obtenir un poste non pérenne ou non satisfaisant que le sexe, l'âge ou le niveau de diplôme.

Le premier modèle montre que le risque de trouver un emploi qui s'avère précaire est plus important pour les chômeurs non indemnisés et, surtout, quand les emplois appartiennent à certaines FAP, telles que les ouvriers qualifiés du gros œuvre du bâtiment, les ouvriers qualifiés de l'électricité et de l'électronique, les ouvriers qualifiés de la manutention, les représentants commerciaux, par rapport aux employés administratifs d'entreprise (tableau 7, logit 1). Ce risque est moindre pour les personnes traditionnellement moins exposées à la précarité, notamment celles âgées de 35 à 49 ans (par rapport à celles de 25 à 34 ans) et celles ayant un niveau de formation supérieur à $\mathrm{bac}+2$ (par rapport à celles qui possèdent le baccalauréat ou moins).

Le deuxième modèle confirme l'importance des métiers dans l'insatisfaction relative à l'emploi retrouvé : les ouvriers qualifiés de la manutention ont plus de probabilité de maintenir leur recherche d'emploi que les employés administratifs d'entreprise, tandis que les ouvriers qualifiés des industries de process, les vendeurs, les attachés commerciaux et représentants, les bouchers, les cuisiniers, les assistants maternels, les agents de sécurité, les aides-soignants en ont moins (tableau 7, logit 2).

Les autres paramètres qui influent sur l'insatisfaction des anciens chômeurs en reprise d'emploi sont : l'âge (moins de probabilités pour les 16-24 ans et pour les 35-49 ans, par rapport aux personnes âgées de 25 à 34 ans), l'indemnisation du chômage (accroissement des probabilités pour les non-indemnisés) et l'exercice d'activités réduites (accroissement également des probabilités d'insatisfaction pour ceux qui en exerçaient avant leur sortie du chômage).

\section{Obtenir un emploi à temps partiel ou à temps plein : le poids des caractéristiques individuelles et du métier}

Un modèle de régression logistique polytomique (multinomial logit) permet de tester les chances de trouver un emploi à temps plein ou à temps partiel choisi versus un emploi à temps partiel contraint. De nouveau, les caractéristiques individuelles jouent nettement moins que les variables d'emploi : les écarts de probabilité avec la référence sont en effet supérieurs pour tous les métiers significatifs. 
Plus en détail, ce modèle montre que la probabilité d'obtenir un contrat à temps plein plutôt qu'un contrat à temps partiel contraint augmente pour les hommes (par rapport aux femmes) ; elle baisse pour les personnes âgées de plus de 34 ans (par rapport à celles âgées entre 25 et 34 ans), ainsi que pour les peu diplômés (par rapport aux bacheliers). Elle diminue sensiblement $(-15 \%)$ pour les chômeurs non indemnisés (par rapport aux chômeurs indemnisés).

Par rapport aux employés administratifs d'entreprises, elle augmente pour quasiment tous les métiers industriels et diminue pour les métiers tertiaires (elle s'effondre pour les aides à domicile et aides ménagères : $-43,5 \%)$. Les chances d'obtenir un emploi à temps plein dépendent donc fortement du métier. Un résultat important ressort ici : le CDI n'exerce aucune influence sur cette probabilité, par rapport aux autres types de contrats (tableau 7 , logit 3$)$.

Quant à la probabilité d'obtenir un contrat à temps partiel choisi versus un contrat à temps partiel contraint, le modèle montre qu' elle diminue pour les hommes (par rapport aux femmes) et pour les diplômés du supérieur, mais aussi ceux de niveau CAP-BEP (par rapport aux bacheliers) et qu'elle augmente pour les personnes âgées de plus de 34 ans (qui ont souvent des charges familiales), par rapport à la référence.

Hormis les techniciens des services administratifs, comptables et financiers où les chances d'obtenir un temps partiel choisi augmentent, les autres FAP ne sont pas significatives. De même, le métier, l'indemnisation, l'exercice d'activités réduites n'ont aucun effet, pas plus que le type de contrat.

Dans ces estimations, le type de contrat est donc " non significatif ", autant sur les chances d'obtenir un contrat à temps plein qu'à temps partiel choisi. Ce résultat tend donc d'une part, à relativiser les vertus souvent supposées du CDI sur la qualité de l'emploi qui lui serait associée; d'autre part, à confirmer nos hypothèses sur l'existence de "CDI précaires » à la sortie du chômage, du moins au niveau du temps partiel contraint. 
Tableau 7. Deux modèles de régression logistique (logit 1 et 2) et un modèle de régression polytomique (multinomial logit 3), toutes FAP

\begin{tabular}{|c|c|c|c|c|}
\hline & \multicolumn{4}{|c|}{ Probabilité de référence (en \%) - écarts de probabilité (en pts) } \\
\hline & $\begin{array}{l}\text { Logit } 1 . \\
\text { Emploi « non } \\
\text { pérenne » }\end{array}$ & $\begin{array}{l}\text { Logit } 2 . \\
\text { Emploi « non } \\
\text { satisfaisant » }\end{array}$ & $\begin{array}{l}\text { Logit 3. Temps plein } \\
\text { vs. temps partiel } \\
\text { contraint }\end{array}$ & $\begin{array}{l}\text { Logit 3. Temps partiel } \\
\text { choisi vs. temps partiel } \\
\text { contraint }\end{array}$ \\
\hline Référence & $15,4 \%$ & $36,0 \%$ & $82,7 \%$ & $32,2 \%$ \\
\hline Femme & réf. & réf. & réf. & réf. \\
\hline Homme & $\sim$ & $\sim$ & 8,4 & $-8,0$ \\
\hline Moins de 25 ans & $\sim$ & $\sim$ & $\sim$ & $\sim$ \\
\hline $25-34$ ans & réf. & réf. & réf. & réf. \\
\hline $35-49$ ans & $-4,1$ & $-6,3$ & $\sim$ & 13,2 \\
\hline 50 ans et plus & $\sim$ & $\sim$ & $\sim$ & 12,5 \\
\hline $\mathrm{Bac}+2$ et plus & $-3,7$ & $\sim$ & $\sim$ & $\sim$ \\
\hline Bac & réf. & réf. & réf. & réf. \\
\hline $\begin{array}{l}\text { CAP (Certificat d'aptitudes } \\
\text { professionnelles) - BEP (Brevet } \\
\text { d'études professionnelles) }\end{array}$ & $\sim$ & $\sim$ & $-6,0$ & $-9,3$ \\
\hline $\begin{array}{l}1^{\text {er }} \text { cycle collège, CEP (Certificat } \\
\text { d'études primaires) }\end{array}$ & $\sim$ & $\sim$ & $-7,2$ & $\sim$ \\
\hline CDI (Contrat à durée indéterminée) & - & - & $\sim$ & $\sim$ \\
\hline $\begin{array}{l}\text { Autre type de contrat et } \\
\text { indépendants }\end{array}$ & - & - & réf. & réf. \\
\hline Non indemnisé & 9,2 & 11,2 & $-14,9$ & $\sim$ \\
\hline Indemnisé & réf. & réf. & réf. & réf. \\
\hline Activités réduites exercées & $-3,0$ & 6,5 & $\sim$ & $\sim$ \\
\hline Absence d'activités réduites & réf. & réf. & réf. & réf. \\
\hline Maraîchers, jardiniers, viticulteurs & $\sim$ & $\sim$ & $-16,7$ & $\sim$ \\
\hline $\begin{array}{l}\text { Ouvriers qualifiés du gros œuvre du } \\
\text { bâtiment }\end{array}$ & 12,5 & $\sim$ & 12,1 & $\sim$ \\
\hline $\begin{array}{l}\text { Ouvriers qualifiés du bâtiment, } \\
\text { second œuvre }\end{array}$ & $\sim$ & $\sim$ & 8,5 & $\sim$ \\
\hline $\begin{array}{l}\text { Ouvriers qualifiés de l'électricité et } \\
\text { électronique }\end{array}$ & $\sim$ & $\sim$ & 15,5 & $\sim$ \\
\hline $\begin{array}{l}\text { Ouvriers qualifiés des industries de } \\
\text { process }\end{array}$ & $\sim$ & $\sim$ & 10,4 & $\sim$ \\
\hline $\begin{array}{l}\text { Techniciens et agents de maîtrise de } \\
\text { la maintenance et de l'organisation }\end{array}$ & $\sim$ & $\sim$ & $\sim$ & $\sim$ \\
\hline Ouvriers qualifiés de la manutention & 18,2 & $\sim$ & $\sim$ & $\sim$ \\
\hline Conducteurs de véhicules & $\sim$ & $\sim$ & $\sim$ & $\sim$ \\
\hline Secrétaires & $\sim$ & $\sim$ & $-16,6$ & $\sim$ \\
\hline Employés de la comptabilité & $\sim$ & $\sim$ & $\sim$ & $\sim$ \\
\hline Employés administratifs d'entreprise & réf. & réf. & réf. & réf. \\
\hline $\begin{array}{l}\text { Techniciens des services } \\
\text { administratifs, comptables et } \\
\text { financiers }\end{array}$ & $\sim$ & $\sim$ & $\sim$ & $\sim$ \\
\hline
\end{tabular}




\begin{tabular}{|l|c|c||c|c|}
\hline \multicolumn{5}{|c|}{ Suite Tableau 7 } \\
\hline Caissiers, employés de libre service & $\sim$ & $\sim$ & $\sim$ & $\sim$ \\
\hline Vendeurs & $\sim$ & $-7,8$ & $\sim$ & $\sim$ \\
\hline $\begin{array}{l}\text { Attachés commerciaux et } \\
\text { représentants }\end{array}$ & 11,0 & $-16,2$ & 13,4 & $\sim$ \\
\hline Bouchers, charcutiers, boulangers & $\sim$ & $-18,6$ & 13,2 & $\sim$ \\
\hline Cuisiniers & $\sim$ & $-10,8$ & $\sim$ & $\sim$ \\
\hline $\begin{array}{l}\text { Employés. agents de maîtrise } \\
\text { hôtellerie-restauration }\end{array}$ & $\sim$ & $\sim$ & $\sim$ & $\sim$ \\
\hline Aides à domicile et aides ménagères & $\sim$ & $\sim$ & $-43,5$ & $\sim$ \\
\hline Assistants maternels & $-8,1$ & $\sim$ & $\sim$ & $\sim$ \\
\hline $\begin{array}{l}\text { Agents de gardiennage et de } \\
\text { sécurité }\end{array}$ & $\sim$ & $-14,0$ & $\sim$ & $\sim$ \\
\hline Agents d'entretien & $\sim$ & $\sim$ & $-18,9$ & $\sim$ \\
\hline Aides-soignants & $\sim$ & $\sim$ & $\sim$ & $\sim$ \\
\hline $\begin{array}{l}\text { Prof. action socioculturelle et } \\
\text { sportive }\end{array}$ & $-7,3$ & $\sim$ & $-18,5$ & $\sim$ \\
\hline
\end{tabular}

Lecture : logit 1. Par rapport aux personnes âgées de 25 à 34 ans, celles âgées de 35 à 49 ans ont moins de probabilité de trouver un emploi non pérenne $(-4,1$ points par rapport à la référence) ;

logit 2. Par rapport aux personnes âgées de 25 à 34 ans, celles âgées de 35 à 49 ans ont moins de probabilités de trouver un emploi non satisfaisant (-6,3 points par rapport à la référence) ;

Logit 3 (multinomial). Par rapport aux femmes, les hommes ont plus de chances de trouver un emploi à temps plein plutôt qu'un emploi à temps partiel contraint (8,4 points par rapport à la référence) ; ils ont moins de probabilités de trouver un emploi à temps partiel choisi plutôt qu'un emploi à temps partiel contraint (-8 points par rapport à la référence).

Significativité : résultats significatifs au moins à $5 \%$.

Champ : 3408 anciens chômeurs ayant trouvé un emploi dans les FAP retenues (sur les 4514 sortants au total en reprise d'emploi, y.c. indépendants).

Source : Enquête Sortants, 2009.

\section{Conclusion}

Au total, les sorties du chômage en 2009 se réalisent massivement vers certains métiers du tertiaire où parfois les rotations de main-d'œuvre ont été importantes. Il s'agit des employés administratifs, des caissiers-employés de libre service, des employés et agents de maîtrise de l'hôtellerie et de la restauration, des agents d'entretien, des aides soignants et des professionnels de l'action sociale notamment. Dans certaines familles professionnelles, les anciens chômeurs accèdent relativement souvent à un emploi en CDI.

Certains CDI sont bien sûr proches de la norme d'emploi (à temps plein, relativement bien rémunérés, satisfaction des personnes) : c'est le cas des techniciens et agents de maîtrise de la maintenance et de l'organisation, des techniciens des services administratifs, comptables et financiers ou des ouvriers qualifiés du bâtiment.

Dans d'autres cas, il s'agit de métiers qui connaissent des taux de rotation très forts (cuisiniers par exemple). Le CDI constitue alors un moyen d'attirer des candidats sur des métiers en tension. Dans d'autres situations enfin, les emplois à temps partiel contraint sont fréquents (employés et agents de maîtrise de l'hôtellerie et de la restauration, caissiers et employés de libre service, aides à domicile et agents d'entretien). 
Le fort taux de CDI parmi les emplois retrouvés n'est donc pas toujours un gage de stabilité ou de qualité de l'emploi. En raison des caractéristiques défavorables de ces CDI et de l'insatisfaction déclarée par les salariés, le turn-over est élevé dans certains métiers.

À la sortie du chômage, la " précarité de droit ", liée au contrat de travail temporaire, est donc associée à une " précarité de fait ", déjà mise en évidence par Fourcade (1992). Notre estimation de la place des CDI non pérennes est nettement supérieure à celle établie par Rouxel (2009), ce qui laisse supposer une accentuation de ce phénomène à la sortie du chômage. Cette précarité " de fait ", qui touche aussi les personnes en CDI, semble actuellement se diffuser autour de certains types de contrats, les CDI à temps partiel ou les CDI intermittents notamment ${ }^{10}$.

Les mobilités externes étudiées ici à la sortie du chômage laissent apparaître différentes facettes de la précarité que génère l'hétérogénéité des CDI. Ces résultats invitent donc à relativiser la stabilité de la relation d'emploi associée au CDI pour les entrants sur le marché du travail, hypothèses confortées par ailleurs dans les travaux de certains juristes (Reynes, $\left.2012^{11}\right)$. La segmentation des emplois ressort ici avec une certaine acuité et l'entrée par « le métier " s'avère donc pertinente ; en effet, globalement, celui-ci joue plus sur les chances d'obtenir un CDI à temps plein ou à temps partiel choisi que les caractéristiques individuelles. Cette importance du métier, et par là même de l'emploi, confirme aussi la pertinence du cadre théorique de la file d'attente (Thurow, 1975).

Certes, ces données individuelles ne permettent pas d'explorer plus avant les modes de gestion différenciés de la main-d'œuvre par les entreprises ou selon les secteurs. Elles montrent toutefois comment certains critères de sélection à l'embauche sont utilisés par les employeurs - le sexe, l'âge ou le diplôme notamment - et quels sont les types de contrat de travail qui sont proposés aux chômeurs. Nos résultats indiquent que ces critères sont mobilisés de manière différenciée selon les métiers. Ces premiers résultats ne fournissent qu'une photographie de la situation des sortants du chômage en 2009. Ils mériteraient d'être prolongés afin de mieux analyser les différents types de CDI qui coexistent sur le marché du travail.

10. Le CDI-I ou contrat à durée indéterminée intermittent est un contrat de travail où le salarié alterne des périodes travaillées et non travaillées; sa rémunération est lissée sur l'année. Il s'agit d'une sorte de contrat à temps partiel annualisé, extrêmement flexible. Il ne garantit aucune durée minimale de travail et peut avoir pour effet de ne faire travailler le salarié que quelques semaines ou quelques mois dans l'année. Le CDI-I ne permet pas au salarié d'obtenir la prime de précarité de $10 \%$, comme pour les CDD et les périodes non travaillées n'ouvrent pas droit à l'assurance chômage. Ce type de contrat existe déjà, mais à condition d'être autorisé par un accord de branche qui pose des limites. L'ANI du 11 janvier 2013 a ouvert, à titre expérimental, la possibilité de recourir directement au CDI intermittent sans accord collectif préalable pour les trois branches suivantes : les organismes de formation, le commerce des articles de sport, équipements de loisirs et les chocolatiers (source: Projet de loi relatifà la sécurisation de l'emploi, Étude d'impact, 5 mars 2013, partie V, République française, p. 145).

11. Du point de vue du droit de l'emploi, cet auteur précise (p. 16) : "Or, si les emplois stables sont pourvus essentiellement par des CDI, le rapport de causalité avec les prescriptions juridiques demeure incertain et le régime normatif des contrats ne saurait apparaître comme le seul facteur explicatif." 


\section{- Bibliographie}

Aeberhardt R., Marbot C. (2010), «Évolution de l'instabilité sur le marché du travail français au cours des trente dernières années ", Courrier des statistiques, $\mathrm{n}^{\circ} 129$.

Amossé T. (2004), "Vingt-cinq ans de transformation des mobilités sur le marché du travail », INSEE, Données sociales, pp. 235-242.

Amossé T., Ben Halima M.-A. (2010), "Mobilité et stabilité sur le marché du travail ", Connaissance de l'emploi, CEE, $n^{\circ} 75$.

Atkinson T. (1984) "Manpower Strategies for Flexible Organisations", Personnel Management, August, pp. 28-31.

Averitt R. (1987), "The dual economy 20 years later", Journal of Economic Issues, 21, 2 , pp. $795-802$.

Bessière S., Omalek L., Viney X., Klein T., Lainé F., Bonnnet A., Zanda J.-L. (2010), "Les métiers en 2009 : seuls quelques métiers résistent à la dégradation du marché du travail », Dares / Centre d'Analyse stratégique, Dares analyses, nº 069.

Bruyère M., Lizé L. (2010), "Emploi et sécurité des trajectoires professionnelles ", Économie et Statistique, no 431-432, pp. 95-113.

Chardon O., Estrade M.-A. (2007), « Les métiers en 2015 », Rapport du CAS-Dares, La Documentation française, Paris.

Doeringer P.B., Piore M. (1971), Internal Labor Markets and Manpower Analysis, Heath Lexington Books, $2^{\circ}$ éd. augmentée, 1985, New York, Armonk Sharpe.

Duhautois R., Petit H., Remillon D. (2012), La mobilité professionnelle, Repères, La Découverte, Paris.

Dupray A. (2005), «Les mobilités en début de vie professionnelle : externes ou internes au entreprises, des évolutions aux effets très différents ", Céreq, Bref, n 216.

Eyraud F., Marsden D. et Silvestre J.-J. (1990), « Marché professionnel et marché interne du travail en Grande-Bretagne et en France ", Revue Internationale du Travail, vol. 129, $\mathrm{n}^{\circ} 4$, pp. 551-569.

Fitoussi J.-P., Laurent É., Maurice J. (2004), Ségrégation urbaine et intégration sociale, Conseil d'Analyse économique, rapport du CAE, nº 45.

Fourcade B. (1992), «L'évolution des situations particulières d'emploi de 1945 à 1990 ", Travail et Emploi, $\mathrm{n}^{\circ}$ 52, pp. 4-19.

Gadrey J. (1992), L'économie des services, Repères, La Découverte.

Gazier B. (2010), Les stratégies des ressources humaines, Paris, La Découverte, col. « Repères ». 
Givord P. (2005), "Formes particulières d'emploi et insertion des jeunes ", Économie et Statistique, $\mathrm{n}^{\circ} 388-389$.

Lainé F. (2010), « La mobilité professionnelle : facteurs structurels et spécificités de l'Îlede-France », Économie et Statistique, n 431-432, pp. 37-56.

Lizé L., Prokovas N. (2009), "La sortie du chômage : le jeu des mobilités ascendantes et descendantes ", Économie Appliquée, tome LXII, n 1, pp. 104-132.

Lizé L., Prokovas N. (2012), «Le risque d'occuper un emploi de mauvaise qualité à la sortie du chômage ", Documents de travail du Centre d'économie de la Sorbonne, n 2012.73.

Marsden D. (1989), Marché du travail : limites sociales des nouvelles théories, Economica.

Marsden D. (1999), A theory of employment systems: micro-foundations of societal diversity, Oxford Press (New York).

Picart C. (2008), «Flux d'emploi et de main-d'œuvre en France : un réexamen », Économie et Statistique, $\mathrm{n}^{\circ}$ 412, pp. 27-56.

Piore M. (1978), « Dualism in the Labor market: A reponse to Uncertainty and Flux, Case of France, Revue économique, vol. 29, n 1, pp. 26-48.

Reynès B. (2011), «Emploi durable et formes d'emploi : la primauté du contrat de travail à durée indéterminée (mythe ou réalité ?) ", La Semaine sociale Lamy (supplément), $\mathrm{n}^{\circ} 1485$, pp. 14-17.

Rouxel C. (2009), "Conditions de travail et précarité de l'emploi », Dares, Premières Synthèses, $\mathrm{n}^{\circ} 28.2$.

Simonnet V., Ulrich V. (2009), «La mobilité entre les métiers », Premières Synthèses, Dares, $n^{\circ}$ 5-3.

Thurow L. C. (1975), Generating Inequality, Basic Books, New York. 